\title{
A modification of line Hausdorff distance for face recognition to reduce computational cost
}

\author{
Dang Nguyen Chau, Do Hong Tuan
}

\begin{abstract}
Face recognition, that has a lot of applications in modern life, is still an attractive research for pattern recognition community. Due to the similarity of human faces, face recognition presents a significant challenge for pattern recognition researchers. Hausdorff distance is an efficient parameter for measuring the similarity between objects. Line Hausdorff distance (LHD) technique, which is the applying of Hausdorff distance for face recognition, gives high accuracy in comparing with common methods for face recognition. For fast screen techniques such as LHD, the computational cost is a key issue. A modified Line Hausdorff distance (MLHD) is proposed in this paper. The performance of the proposed method is compared with LHD method for face recognition in various conditions: 1) ideal condition of face, 2) varying lighting conditions, 3) varying poses and 4) varying face expression. It is very encouraging that the proposed method gives lower computational cost than LHD while keeping the accuracy of face recognition equal to the LHD method.
\end{abstract}

Index Terms - Face cognition, Line Hausdorff Distance, Hausdorff Distance, Modified Line Hausdorff Distance.

\section{INTRODUCTION}

A utomatic face recognition is an active research area and has had a lot of publications in last two decade years. Face recognition has lot of applications in modern life such as bank card identification, access control, 'mug shot' searching, security monitoring systems.

Face recognition is used for identification one or more persons from a still image or a video by

Manuscript Received on March 15 ${ }^{\text {th }}$, 2017, Manuscript Revised on November 01 ${ }^{\text {st }}, 2017$.

Dang Nguyen Chau, Ho Chi Minh City University of Technology - VNU-HCM, Hochiminh City, Vietnam (e-mail: chaudn@hcmut.edu.vn).

Do Hong Tuan, Ho Chi Minh City University of Technology - VNU-HCM, Ho Chi Minh City, Vietnam (email: do-hong@hcmut.edu.vn). comparing the input image with face images stored in database. Face features of images in database are extracted and stored offline. The same features of the input image are extracted too and comparing with the features of each model image in the database. Due to the similarity of human face, comparing techniques of face features still presents significant challenge for pattern recognition researchers. There were a lot of methods for face recognition have been proposed. However, there was no method is the best in comparing with other methods [1]. Line Hausdorff Distance (LHD), which proposed by Gao and Leung [2], gives high accuracy for face recognition in comparing with other common methods for face recognition. Moreover, LHD is also stable in various condition of face recognition such as: lighting conditions, varying pose conditions and varying face expressions. This makes LHD become more attractive for applying for face recognition applications, where the real lighting conditions, poses and face expressions are different from image with ideal conditions in database.

In most face recognition systems, searching the best matching face in database is the most computational expensive operation due to the large number of images in the database. Efficient search method is more attractive for a face recognition system. The proposed method in this paper, Modified Line Hausdorff Distance (MLHD), give lower computational cost than LHD method while the accuracy for face recognition is equal to the LHD method. In the rest of paper, the review of LHD method is presented in section 2. The proposed method, MLHD, is continued in section 3. Section 4 is the comparing recognition rate of the proposed method MLHD with LHD method. The paper is closed in section 5 with some comments. 


\section{Line HaUSDORFF DisTANCE}

The edges of an face image, which is the reflecting of large local intensity changes caused by the geometrical structure of the face, are the important features of human face. However, edge of an human face image has not used for face recognition until Takács [3]. Takács [3] and Gao [4], used Haudorff distance for matching dominant points of the edge map of a face image and called Modified Hausdorff Distance (MHD). Gao and Leung [2] also used Hausdorff distance for matching face image but using the lines, which is the connection of dominant points, of the edge map, called Line Hausdorff Distance (LHD).

Edge map of an face image is the map of dominant points of edge curve of face image. For generating an edge map, first, edge curve of the face image is extracted. There are a lot of edge extraction methods proposed. However, Heath et al. [5] showed that "no one single edge detector was best overall; for any given image it is difficult to predict which edge detector will be best". For generating the edge map of face image, we use the edge detector which is proposed by Babu [6] followed by a thinning process for one pixel width edge curve extraction. The Dynamic two strip (Dyn2S) [7] is applied on edge curve for generating the dominant points of the edge curve. An example of an edge map of a face image is shown in Fig. 1.

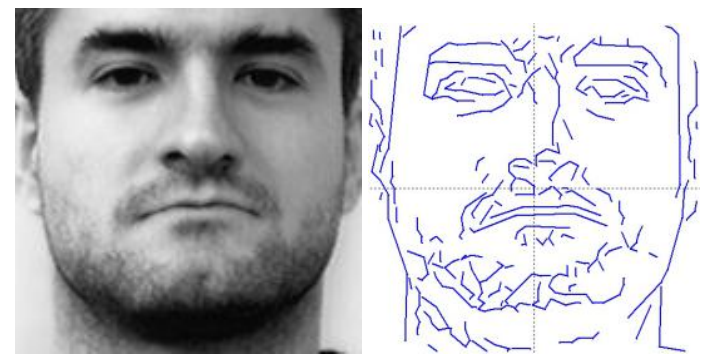

Fig. 1. A person face and its edge map.

LHD uses Hausdorff distance for measuring the distance between lines in edge map (LEM), which are the connection between dominant points. Given two LEMs $M^{l}=\left\{m_{1}^{l}, m_{2}^{l}, . . m_{p}^{l}\right\}$ (representing the model LEM in database) and $T^{l}=\left\{t_{1}^{l}, t_{2}^{l}, . . t_{q}^{l}\right\} \quad$ (representing the test image LEM), the notation $l$ means line, for discriminating from the dominant point used in MHD. The distance between two lines is defined as

$$
d\left(m_{i}^{l}, t_{j}^{l}\right)=\sqrt{d_{o}^{2}\left(m_{i}^{l}, t_{j}^{l}\right)+d_{p a}^{2}\left(m_{i}^{l}, t_{j}^{l}\right)+d_{p e}^{2}\left(m_{i}^{l}, t_{j}^{l}\right)}
$$

where $d_{o}$ is the orientation distance is defined as

$$
d_{o}\left(m_{i}^{l}, t_{j}^{l}\right)=\theta^{2}\left(m_{i}^{l}, t_{j}^{l}\right) / W
$$

$\theta$ is the smallest intersecting angle between two lines, $W$ is a parameter determined by a training process. $d_{p a}$ and $d_{p e}$ are the parallel distance and perpendicular distance between two lines, could be calculated as

$$
\begin{aligned}
& d_{p a}\left(m_{i}^{l}, t_{j}^{l}\right)=\min \left(l_{p a 1}, l_{p a 2}\right) \\
& d_{p e}\left(m_{i}^{l}, t_{j}^{l}\right)=l_{p e}
\end{aligned}
$$

The parallel distance and perpendicular distance as Eq. (3) and Eq. (4) is used for two parallel lines as Fig. 2.

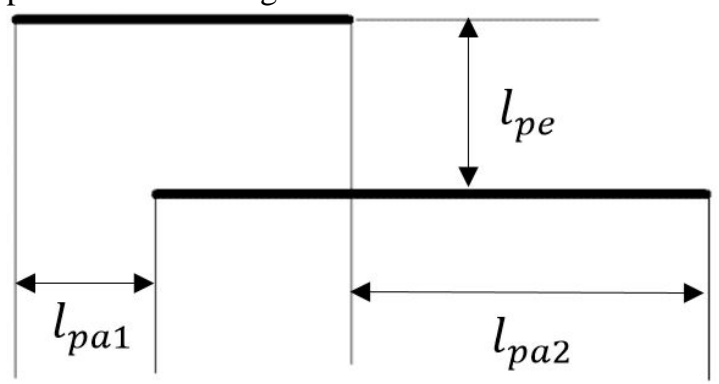

Fig. 2. Distance between two parallel lines.

In general, two lines are not parallel, so we must rotate the shorter line with its midpoint as rotation center before calculating $d_{p a}$ and $d_{p e}$. The reason of choosing shorter line for rotating because this would cause less distortion to the original line pair. In order to reducing the effect of the broken line caused by the segmentation error of the missing or adding of dominant points, the parallel distance will be zero if one line is within the range of other as Fig. 3. 


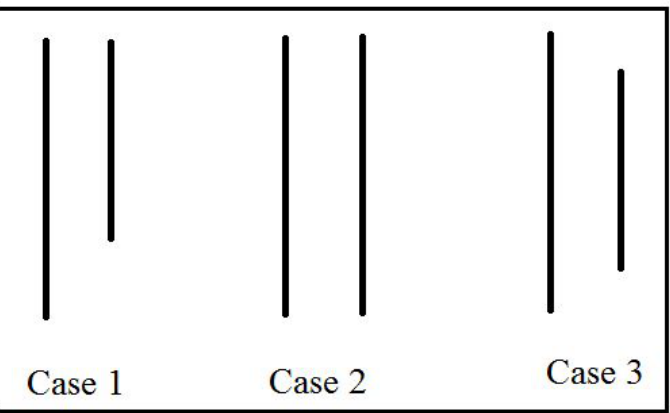

Fig. 3. Distance between two parallel lines

A primary line Hausdorff distance for measuring the similarity between lines could be defined as

$$
H_{p L H D}\left(T^{l}, M^{l}\right)=\max \left(h\left(M^{l}, T^{l}\right), h\left(T^{l}, M^{l}\right)\right)
$$

where

$$
h\left(M^{l}, T^{l}\right)=\frac{1}{\sum_{m_{i}^{l} \in M^{l}} l_{m_{i}^{l}}^{l}} \sum_{m_{i}^{l} \in M^{l}} l m_{i}^{l} \cdot \min _{t_{j}^{l} \in T^{l}} d\left(m_{i}^{l}, t_{j}^{l}\right)
$$

and $l_{m_{i}^{l}}$ is the length of the line $m_{i}^{l} \cdot h\left(M^{l}, T^{l}\right)$ is called the direct Hausdorff distance between two LEMs.

However, the Hausdorff distance as Eq. 6 still has weakness. Supporting $T$ is the LEM of the test image and $t_{j}$ is a line in $T ; M_{c}$ and $M_{n}$ are the corresponding identical and nonidentical model of the test image in the database. If the corresponding line of $t_{j}$, line $m_{c}$, in $M_{c}$ is missing because of segmentation error, the direct Hausdorff distance as Eq. (6) between $T$ and $M_{c}$ will use $d\left(m_{c n}^{l}, t_{j}^{l}\right)$ for calculating. $d\left(m_{c n}^{l}, t_{j}^{l}\right)$ is the distance between line $t_{j}$ to the closest line $m_{c n}$ in corresponding LEM $M_{c}$, however, different from the corresponding line $m_{c}$. Similarity, the direct Hausdorff distance between $T$ and $M_{n}$ will use $d\left(m_{n n}^{l}, t_{j}^{l}\right)$ for calculating, where $m_{n n}$ is the closet line of $t_{j}$ in the LEM $M_{n}$. It is possible the $d\left(m_{c n}^{l}, t_{j}^{l}\right)$ is very large than $d\left(m_{n n}^{l}, t_{j}^{l}\right)$ and $T$ will take the $M_{n}$ as it matching LEM in the database. Though $d\left(m_{c n}^{l}, t_{j}^{l}\right)$ and $d\left(m_{n n}^{l}, t_{j}^{l}\right)$ are not the corresponding lines of $t_{j}$, they make $M_{n}$ more similar to $T$ than $M_{c}$ and this cause the mismatch.

Gao and Leung suggested using the number of corresponding pair between test and model image in the measure of the similarity. Assume that line $t_{j}$ of the test LEM take $m_{i}$ of the model LEM as closet line. They defined $N_{P}$ and $N_{A}$ are the position and angle neighbor. If the line $m_{i}$ is in range of $N_{P}$ and $N_{A}$, the line $m_{i}$ might be the corresponding line of line $t_{j}$. Such line $t_{j}$ is called the high confident line. A ratio of confident, which is the ratio between the number of high confident line $\left(N_{h c}\right)$ and number of line the LEM $\left(N_{\text {total }}\right)$, is defined as

$$
R=\frac{N_{h c}}{N_{\text {total }}}
$$

Hence, the complete version of LHD will become

$$
H_{L H D}\left(T^{l}, M^{l}\right)=\sqrt{H_{p L H D}^{2}\left(T^{l}, M^{l}\right)+\left(W_{n}\left(1-\frac{R_{M}+R_{T}}{2}\right)\right)^{2}}
$$

where $R_{M}$ and $R_{T}$ are the ratio of confident of input and model LEM, $W_{n}$ is a parameter that is determined in training process. In the experiment, we use the Bern university database [8] for training process. The parameter of LHD are obtained as follow: $N_{P}=6, N_{A}=30, W=30$, $W_{n}=5$.

\section{Modified Line HausdorfF Distance (MLHD)}

In the calculating of the $h\left(M^{l}, T^{l}\right)$ as Eq. (6), for each line $m_{i}$ in model LEM $M$, we have to calculate the distance between two lines $d\left(m_{i}^{l}, t_{j}^{l}\right)$ for all lines $t_{j}$ in the test LEM. After that, we find the line $t_{j}$, which has the minimum distance $d\left(m_{i}^{l}, t_{j}^{l}\right)$. This is the degree of redundancy of LHD method.

The line $t_{j}$, which has the minimum distance to the line $m_{i}$, might be the corresponding line of line $m_{i}$ in the model edge map. Such corresponding line could not be too far from the line $m_{i}$ of the model edge map. From this idea, we see that for finding the line in test edge map 
has minimum distance to a line $m_{i}$ in model edge map, we could find from the lines, which have the distance to line $m_{i}$ is $d_{c}$ lose.

Suppose matrix $M$ and $T$ are the LEM of model and test image. Each line of matrix is the coordinates of two end-points of each line in the LEM. The Algorithm 1 shows the proposed idea for calculating $h\left(M^{l}, T^{l}\right)$. In the Algorithm 3, the parameter $D$ is the size of the image, $\mathrm{Kcg}$ is a parameter that we could find from training process. $T_{m p 1}$ and $T_{m p 2}$ are the coordinates of the midpoint of each line in the edge map, which could be find from the coordinates of two endpoints of each line.

Algorithm 1 : The proposed idea

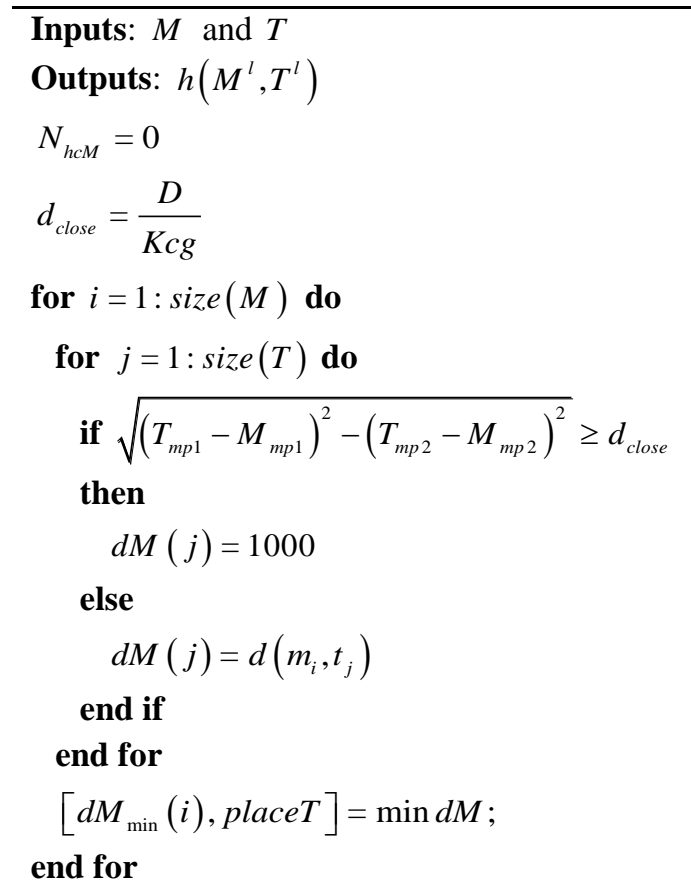

If we call $P$ and $Q$ are the number of lines in the model and test LEM, the computational complexities of LHD will be $O(k P Q)$, where $k$ is the time for calculating distance between two line $d\left(m_{i}^{l}, t_{j}^{l}\right)$. With the proposed idea, the computation complexities of MLHD will be the same as LHD method but reducing $K c g$ time.

\section{EXPERIMENT RESULT}

In this paper, we investigate the system performance of MLHD for face recognition under: 1) ideal lighting condition and pose, 2) various lighting condition and 3) varying poses. Two common face databases are used. Bern University database [8] contains 300 images of 10 persons, each person has 10 pictures (2 pictures of frontal face, 2 pictures with looking right face, 2 pictures with looking left face, 2 pictures with looking up face and 2 pictures with looking down face). AR face database [9] contains 2600 pictures of 100 persons (50 men and 50 women), each person has 26 pictures with first 13 pictures has various lighting condition, face expression and last 13 pictures is the same as first 13 pictures with 2 weeks later taking. In the experiment, all picture is resize at $160 \times 160$ pixels.

\subsection{The effect of $\mathrm{Kcg}$}

In this experiment, we use frontal face image of Bern database as the training set. There are 30 frontal face images of 30 person for the database and other 30 frontal face images as test images. The recognition result is shown in Fig. 4.

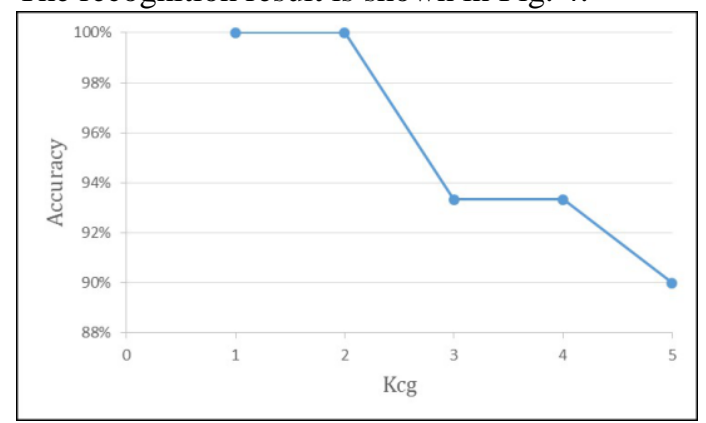

Fig. 4. Effect of $\mathrm{Kcg}$ on system performance

From the result for right recognition for frontal face image of Bern database, we choose $K c g=2$ for all other experiments in this paper. The experiment was conducted on a PC station with $3.7 \mathrm{GHz}$ CPU and $2 \mathrm{~GB}$ RAM. The matching time for BERN database is shown in Table 1. The proposed algorithm has matching time $40 \%$ less than the original LHD.

TABLE 1

MATCHING TIME FOR BERN DATABASE

\begin{tabular}{ccc}
\hline \multicolumn{3}{c}{ MATCHING TIME FOR BERN DATABASE } \\
\hline $\begin{array}{c}\text { Matching time } \\
\text { (second) }\end{array}$ & 337 & MLHD \\
\hline \hline
\end{tabular}

In general, the lines of face's LEMs is equally contributing in the face. So the matching time for proposed algorithm will be a half of the LHD algorithm.

\subsection{Face recognition under ideal condition}

In this experiment, we use a pair of frontal face, which is the ideal conditions picture, of each 
person in the Bern database and AR database for face recognition. With Bern database, we have 30 images for database and other 30 images as the input. With the AR database, we have 100 images for database and 100 other images for the input. Recognition rate of LHD and MLHD method is shown in Table 2.

TABLE 2

RECOGNITION RATE FOR IDEAL CONDITION

\begin{tabular}{ccc}
\hline \hline Method & Bern & AR \\
\hline LHD & $100 \%$ & $93 \%$ \\
MLHD & $100 \%$ & $90 \%$ \\
\hline
\end{tabular}

With Bern University database, all methods give correct recognition of $100 \%$ because there are minor differences between two frontal face pictures. However, two frontal face pictures of AR database is taken within 2 weeks, this makes large difference between two pictures.

Here we see that the proposed method MLHD gives lower accuracy than LHD method for face recognition. However, the proposed method has computational complexities is a half in comparing with LHD method.

\subsection{Face recognition under varying lighting conditions}

In this experiment, 100 frontal face pictures of AR database are used as the database. Each person has 2 pictures with the left-light, 2 pictures with the right-light and 2 pictures with the bothlight. So with each lighting condition, we have 200 pictures as the input. The face recognition under varying lighting condition result is showed in Table 3.

TABLE 3

RECOGNITION RATE UNDER VARYING LIGHTING CONDITIONS

\begin{tabular}{ccc}
\hline \hline Method & LHD & MLHD \\
\hline Left light on & $93 \%$ & $92 \%$ \\
Right light on & $87 \%$ & $89 \%$ \\
Both light on & $71 \%$ & $71 \%$ \\
\hline \hline
\end{tabular}

In all conditions of the light, MLHD method archives equal recognition rate to the LHD method. This means MLHD method is stable with lighting conditions of the image as LHD method. This makes MLHD method, also LHD method, become more attractive with real applications, where the lighting conditions is not controlled, than common face recognition method as Eigenface.

\subsection{Face recognition with varying poses}

In this experiment, 30 frontal face pictures of Bern database are used as the database. With each person, we use one image of looking left, looking right, looking up and looking down. So we have 30 images as in put for each condition of pose. The face recognition with varying poses result is showed in Table 4.

TABLE 4

RECOGNITION RATE WITH VARYING POSES

\begin{tabular}{ccc}
\hline \hline Method & LHD & MLHD \\
\hline Looking left & $47 \%$ & $53.33 \%$ \\
Looking right & $54 \%$ & $50 \%$ \\
Looking up & $69 \%$ & $70 \%$ \\
Looking down & $62 \%$ & $56.67 \%$ \\
Avarage & $58 \%$ & $57.5 \%$ \\
\hline \hline
\end{tabular}

The proposed algorithm, MLHD, gives higher accuracy for looking left and looking up of model's face while lower accuracy for others case of facing poses. In the average, the proposed algorithm give the same result as the LHD algorithm.

\subsection{Face recognition with varying face expression}

In this experiment, 100 frontal face pictures of AR database are used as the database. Each person has 2 pictures with each face expression: smiling, angry and screaming. The face recognition with varying face expression result is showed in Table 5.

TABLE 5

RECOGNITION RATE WITH VARYING POSES

\begin{tabular}{ccc}
\hline \hline $\begin{array}{c}\text { Face } \\
\text { expression }\end{array}$ & LHD & MLHD \\
\hline Smiling & $47 \%$ & $53.33 \%$ \\
Angry & $54 \%$ & $50 \%$ \\
Sceaming & $69 \%$ & $70 \%$ \\
\hline \hline
\end{tabular}

The original LHD gives high accuracy for face recognition with smiling and angry expression of human face, however, low accuracy for screaming expression. The proposed algorithm gives slightly lower accuracy for smiling and angry expression than original LHD. However, with screaming expression, the proposed algorithm gives much higher than original LHD. As the result in Table 5 , the proposed algorithm is more stable than original LHD for face recognition with varying face expressions.

\section{CONCLUSION}

LHD method is an advantage method for face 
recognition in comparing with Eigenface, which is the most common method for face cognition used by most researchers of face recognition community. Beside getting higher recognition rate than Eigenface method, LHD method also shows that it is stable with various lighting conditions, varying poses and varying face expression.

This paper has proposed MLHD method. MLHD method gives the same recognition rate as LHD method while getting lower computational complexities. For $K c g=2$, MLHD method gives a half computational complexities of LHD method. This is valuable which a face recognition application, where the number of image for searching is huge. Beside that, MLHD also shows that it is stable with non-ideal conditions of the image.

\section{REFERENCES}

[1] S. A. Patil and D. P. J. Deore, "Face recognition: A survey", International Journal Informatics Engineering, vol. 1, Dec 2013

[2] Y. Gao, and M.K.H. Leung, "Face Recognition Using Line Edge Map", IEEE Transactions On Pattern Analysis And Machine Intelligence, vol. 24, no. 6, 2002, pp. 764 778.

[3] B. Takács, "Comparing Face Images Using the Modified Hausdorff Distance", Pattern Recognition, vol. 31, 1998, pp. 1873-1881

[4] Y. Gao, "Efficiently comparing face images using a modified Hausdorff distance", IEEE Proc. Vision, Image and Signal Processing, vol. 150, no. 6, Dec. 2003, pp. 346-350.

[5] M. D. Heath, S. Sarkar, T. Sanocki, and K. W. Bowyer, "Comparison of edge detectors: a methodology and initial study”, Comput. Vis. Image Underst., vol. 69, 1998, pp. $38-54$.

[6] R. Nevatia, and K. R. Babu, "Linear feature extraction and description", Comput. Graph. Image Process., vol. 13, pp. 257-269, 1980

[7] M. K. H. Leung and Y. H. Yang, "Dynamic two-strip algorithm in curve fitting", Pattern Recognition, vol. 23, 1990, pp. 69-79.

[8] Bern University Face Database, http://www.fki.inf.unibe.ch/databases/iam-faces-database, 2002.

[9] A. M. Martinez and R. Benavente, "The AR Face Database”, CVC Technical Report \#24, June 1998.
Dang Nguyen Chau received the B.S. degree in electrical engineering from the Ho Chi Minh City University of Technology, VNU-HCM, Vietnam, in 2010 and the M. Eng. degree in electrical, electronic and information engineering from the Toyohashi University of Technology, Japan in 2013. Since 2011 he has been with the Faculty of Electrical and Electronics Engineering, Ho Chi Minh City University of Technology, VNU-HCM, Vietnam. His research interests include iamge processing, computer vision and machine learning.

Do Hong Tuan received the B.S. and M. Eng. degrees in electrical engineering from $\mathrm{Ho}$ Chi Minh City University of Technology, VNU-HCM, Vietnam, in 1994 and 1997, respectively, the M.Sc. and $\mathrm{Ph}$. D. degrees in communication engineering from the Munich University of Technology, Germany, in 2000 and 2004, respectively. Since 1994 he has been with the Faculty of Electrical and Electronics Engineering, Ho Chi Minh City University of Technology, VNU-HCM, Vietnam. His research interests include stochastic signal processing and applications for communications, image and video. 


\title{
Cải tiến tốc độ tính toán cho phương pháp khoảng cách Hausdorff đường dùng cho nhận dạng khuôn mặt
}

\author{
Đặng Nguyên Châu và Đỗ Hồng Tuấn
}

\begin{abstract}
Tóm tắt— Nhận dạng khuôn mặt có rất nhiều ứng dụng trong cuộc sống hiện đại ngày nay và vẫn là một hướng nghiên cứu được quan tâm nhiều của cộng đồng khoa học về nhận dạng. Do sự giống nhau của các khuôn mặt người, nhận dạng khuôn mặt vẫn còn là một thách thức lớn cho các nhà nghiên cứu về nhận dạng để đạt được tỷ lệ nhận dạng chính xác cao. Khoảng cách Hausdorff là một phương pháp nhanh và hiệu quả trong việc nhận dạng khuôn mặt. Phương pháp khoảng cách Hausdorff đường (LHD) là một phương pháp áp dụng khoảng cách Hausdorff trong việc nhận dạng khuôn mặt và cho tỷ lệ nhận dạng chính xác rất cao khi so sánh với các phương pháp phổ biến về nhận dạng khuôn mặt. Vì là một phương pháp dò tìm nhanh nên độ phức tạp tính toán là một điều rất quan trọng. Trong bài báo này chúng tôi đề xuất một cải tiến của phương pháp LHD trong nhận dạng khuôn mặt. Độ chính xác của thuật toán đề xuất sẽ được so sánh với phương pháp LHD trong các điều kiện khác nhau: 1) điều kiện lý tưởng; 2) các điều kiện khác nhau của ánh sáng, 3) các góc chụp khác nhau và 4) các cảm xúc khác nhau của khuôn mặt. Trong tất cả các khảo sát thì phương pháp đề xuất đều cho kết quả nhận dạng chính xác gần như ngang bằng với phương pháp LHD nhưng độ phức tạp tính toán thì thấp hơn phương pháp LHD rất nhiều.
\end{abstract}

Tù khóa — Nhận dạng khuôn mặt; Khoảng cách Hausdorff, khoảng cách Hausdorff đường. 